\title{
Multicolor fluorescence in situ hybridization with combinatorial labeling probes enables a detailed karyotype analysis of Larix principis-rupprechtii
}

\author{
BO LIU ${ }^{1}$, LIWANG QI ${ }^{2}$, RUIYANG CHEN ${ }^{1}$ and WENQIN SONG ${ }^{1 *}$ \\ ${ }^{1}$ Laboratory of Cell and Molecular Biology, College of Life Sciences, Nankai University, Tianjin 300071, \\ China \\ ${ }^{2}$ Laboratory of Cell Biology, The Research Institute of Forestry, Chinese Academy of Forestry, Beijing \\ 100091, China
}

\begin{abstract}
The chromosomes $(2 \mathrm{n}=2 \mathrm{x}=24)$ of Larix principis-rupprechtii are composed of six pairs of large metacentrics and six pairs of medium-sized submetacentrics. The identification of homologous pairs is hampered by their high degree of similarity at the morphological level in each group. As one of the most extensively used methods in molecular cytogenetics producing chromosome landmarks, fluorescence in situ hybridization (FISH) has significantly facilitated karyotype construction, especially in species with morphologically similar chromosomes. This study developed a simple but effective use of combinatorial labeling probes to distinguish chromosomes of Larix principis-rupprechtii by multicolor FISH. Three highly repetitive sequences in Larix were selected: $25 \mathrm{~S}$ rDNA hybridized at all of the secondary constrictions of two pairs of metacentrics and the largest pair of submetacentrics; 5S rDNA hybridized at subtelomeric sites of one pair of metacentrics that also harboured $25 \mathrm{~S}$ rDNA on different arms; LPD family sequences are tandem repeats hybridized at proximal regions of 22 chromosomes. The three different probes were labeled with only two different labels, hybridized to metaphase chromosomes of Larix principis-rupprechtii, simultaneously visualized, and unequivocally distinguished in a single FISH experiment. These multicolor FISH marks largely improved the karyotype analysis of Larix principis-rupprechtii.
\end{abstract}

Key terms: combinatorial labeling probes, fluorescence in situ hybridization, karyotype, Larix principisrupprechtii, LPD repeats, $25 \mathrm{~S}$ and $5 \mathrm{~S}$ rDNA

Larix principis-rupprechtii is a perennial deciduous arbor mainly planted in north of China. The diploid chromosome number $(2 n=2 x=24)$ is very conserved in the family of Pinaceae, with little karyotype differentiation between species (Friesen et al., 2001). The chromosomes of Larix principis-rupprechtii constitute a bimodal karyotype in which they are divided into two groups, a group of twelve large metacentrics and the other group of twelve medium-sized submetacentrics (Zhang et al., 1985). However, in each group the identification of homologous pairs is hampered by similar length and slight differences at the morphological level. On the other hand, chromosome landmarks produced by fluorescence in situ hybridization (FISH) with various repetitive sequences as probes make the identification of chromosomes easier. This strategy has been applied successfully in several species of other genera in Pinaceae, e.g. Pinus (Hizume et al., 2002b; Liu et al., 2003) and Picea (Brown et al., 1998; Siljak-Yakovlev et al., 2002; Vischi et al., 2003). In Larix, an improvement has been proposed by Lubaretz et al. (1996) with rDNA and telomeric repeats probes, but these probes are inadequate for distinguishing every chromosome pair. Hizume et al. (2002a) identified and characterized the LPD family as tandemly repetitive DNA sequences in Larix leptolepis, and they concentrate in

\footnotetext{
*Corresponding author: W. Song, Laboratory of Cell and Molecular Biology, College of Life Sciences, Nankai University, Tianjin 300071, China; e-mail: songwq@nankai.edu.cn; Tel.: +86-22-23508241; Fax: +86-22-23497010
} 
proximal regions of most chromosomes of Larix so that the LPD repeats could be a novel probe in chromosome identification of Larix.

The commercial availability of a range of different fluorophores has enabled the simultaneous imaging of several target sequences in one experiment. In plants, however, traditionally a maximum of three fluorophores are used simultaneously, which emit either blue (amino-methylcoumarin; AMCA), green (fluorescein and derivatives, e.g. FITC) or red light (Texas red, rhodamine, cyanine $3 ; \mathrm{Cy} 3)$. Routine counterstaining of chromosomes with for example DAPI, YOYO or propidium iodide, effectively reduces this number since emission wavelengths of these counterstains interfere with emission wavelengths of the commonly used fluorophores. Combinatorial labeling, which incorporates more than one fluorophore into a probe, is a means by which a greater number of chromosome targets may be discriminated. It was developed for human cytogenetics and is considered as a breakthrough in clinical diagnostics (Ried et al., 1992). In plants, Jiang et al. (1995) successfully mapped three different bacterial artificial chromosomes in the rice genome with this approach and Hasterok et al. (2002) improved this method in Secale cereale and Triticum aestivum. To date, combinatorial labeling of probes has scarcely been used for molecular cytogenetics of gymnosperms.

In this study, we developed a simple and efficient technique of multicolor fluorescence in situ hybridization in Larix principis-rupprechtii, which allows the use of probes labeled with two standard labels to map simultaneously three different DNA sequences along somatic chromosomes. Furthermore, a more detailed karyotype of Larix principis-rupprechtii could be constructed with these landmarks produced by the multicolor FISH.

Seeds of L. principis-rupprechtii were collected in a planted larch forest at Dagujia Forestry Centre in Liaoning Province, China. The seeds were sown in sterilized sand in a pot, and 10-14 days later the primary root tips were collected for chromosomal analysis. The root tips were treated with $0.05 \%$ colchicine at $20^{\circ} \mathrm{C}$ for $14 \mathrm{~h}$, fixed in chilled Carnoy's fixative (ethanol: acetic acid $=3: 1, \mathrm{v} / \mathrm{v}$ ) overnight Fixed root tips were macerated in an enzyme mixture containing 2\% cellulase ("Onozuka" R-10, Kinki Yakult) and 1\% pectolyase (Aspergillus niger, Serva) in citrate/citric acid buffer $(\mathrm{pH} 4.5)$. The meristematic cells were squashed under a coverslip on a glass slide and air dried after the coverslip was removed with liquid nitrogen.

Total genomic DNA was extracted from young leaves of L. principis-rupprechtii by the CTAB method (Murray and Thompson, 1980). A $2.3 \mathrm{~kb}$ subclone of the $25 \mathrm{~S}$ rDNA coding region of Arabidopsis thaliana (Unfried and Gruendler, 1990) was labeled with Biotin-16-dUTP (Roche) by randomlyprimed DNA synthesis according to manufacturer's instructions. The $5 \mathrm{~S}$ rDNA clone pTa794 (Gerlach and Dyer, 1980) was amplified and labeled with digoxigenin (DIG)-11-dUTP (Roche) using PCR with universal M13 primers. The LPD family sequence was amplified from total genomic DNA of L. principis-rupprechtii by PCR using the primers LPD-f: 5' CATGCATCCGGAAATAAGAA-3' and LPD-r: 5'-CGCAAACTATAGAAAGTCGT3' (Hizume et al., 2002a), and the product was purified and labeled the same way as $25 \mathrm{~S}$ rDNA but simultaneously and in equal proportions with digoxigenin-11-dUTP and biotin-16-dUTP.

FISH procedure was carried out as described previously (Liu et al., 2005). Briefly, chromosomal DNA was denatured at $70^{\circ} \mathrm{C}$ for $2 \mathrm{~min}$ in $70 \%$ formamide, $2 \times \mathrm{SSC}$. Probes dissolved in $2 \times \mathrm{SSC}, 5 \%$ dextran sulfate, and $50 \%$ formamide were denatured at $94^{\circ} \mathrm{C}$ for $10 \mathrm{~min}$. The hybridized probes were detected with antiDIG-fluorescent-conjugate (FITC, Roche) and Strepetavidin-Cy3 (Sigma). The slides were counterstained with DAPI (4',6diamidino-2-phenylindole, Sigma) and mounted in Vectashield mounting medium (Vector Laboratory). The hybridization signals were visualized and recorded using Nikon 80i fluorescence microscope and a cooled CCD camera (SPOT, Diagnostic Instruments) equipped with the version 
4.0.8 of the Spot software (Diagnostic Instruments). Ten metaphases were scored. Images were processed using Adobe Photoshop 7.0.

The length of short and long arms was measured, and arm ratio (length of long arm/length of short arm) and relative length were estimated for each chromosome. The nomenclature system for the position of the centromere followed that of Levan et al. (1964). Complementary chromosomes were arranged in order of decreasing length.
Figure 1 shows the results of simultaneous triple FISH to the somatic metaphase chromosomes of L. principisrupprechtii. Figure $1 \mathrm{~A}-\mathrm{C}$ show results from single band pass filters in blue, red and green channels, respectively, whilst Figure $1 \mathrm{D}$ present electronically superimposed images combining the three individual channels. In Figure 1B, 28 distinct red signals with different size and intensity indicated the hybridized loci of biotinlabeled probes, six of which marked the
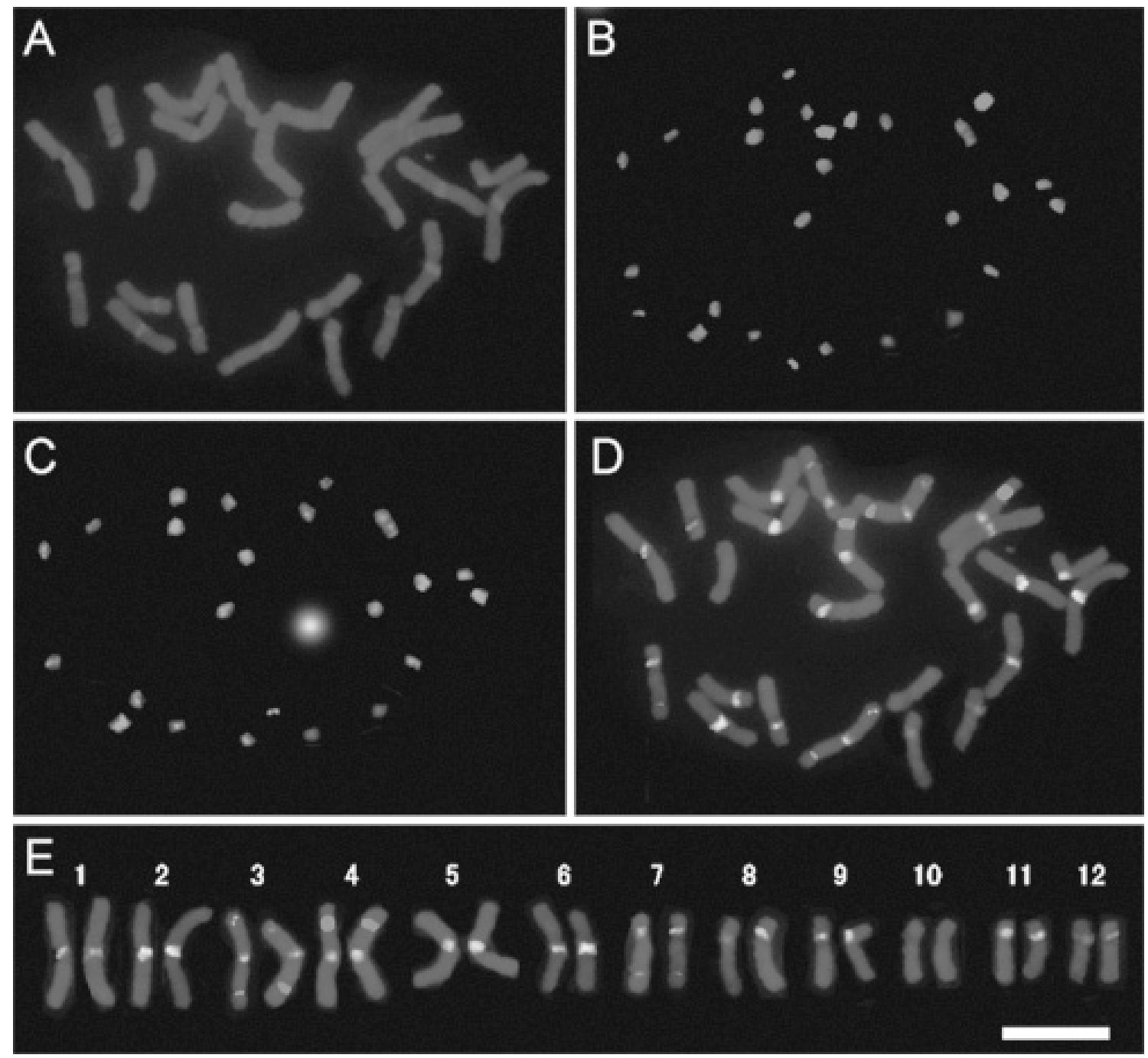

Figure 1: Simultaneous triple FISH to somatic metaphase chromosomes of L. principis-rupprechtii. (A) Chromosome spread counterstaining with DAPI (blue). (B) Red hybridized signals from $25 \mathrm{~S}$ rDNA and LPD repeats. (C) Green hybridized signals from 5S rDNA and LPD repeats. (D) Chromosome spread simultaneously with 25S rDNA, 5S rDNA, and LPD repeats loci in pink, green, and white color, respectively. (E) Homologous chromosome pairing and ordering. Bar. $5 \mu \mathrm{m}$. 
$25 \mathrm{~S}$ rDNA loci and the other 22 were produced by LPD repeats probe. In Figure 1C, 24 distinct green signals indicated the hybridized loci of DIG-labeled probes, two tiny ones represented the 5S rDNA loci and the other 22 produced by LPD repeats probe were the same in size and intensity with the red signals of the same location in Figure 1B. After composed Figure 1A-C, signals with three different color were shown at chromosome spread (Fig 1D). The six pink signals were the $25 \mathrm{~S}$ rDNA red signals against the blue DAPI counterstaining and located at the secondary constrictions of two pairs of metacentrics and the largest pair of submetacentrics. The two tiny green $5 \mathrm{~S}$ rDNA signals located at the subterminal regions of one pair of metacentrics that also harbored a $25 \mathrm{~S}$ rDNA locus on different arms. Since the combinatorial labeling LPD repeats fluoresced red and green simultaneously (Fig 1B and C), they showed white signals in proximal regions of 22 chromosomes in the composite image against the blue DAPI counterstaining of chromosomes. Thus, the multicolor discrimination of three different DNA sequences was accomplished within a single FISH experiment with only two labels.

The chromosomes of L. principisrupprechtii in a given cell can be easily divided into two groups according to their length and centromere positions. Furthermore, with the help of these FISH signals above, we could complete the identification of homologous chromosome pairs within each group easily. First, by the $25 \mathrm{~S}$ and $5 \mathrm{~S}$ rDNA signals, three pairs of chromosomes could be identified unambiguously, as a double marked metacentric pair with both $25 \mathrm{~S}$ and $5 \mathrm{~S}$ rDNA loci on its different arms, a metacentric pair with only $25 \mathrm{~S}$ rDNA loci, and a submetacentric pair with $25 \mathrm{~S}$ rDNA loci on its long arms. Although DAPI bands concentrated in proximal regions of most chromosomes are displayed after DAPI counterstaining (Fig 1A), the white LPD repeats signals representing the same region exclusively (Hizume et al., 2002a) show more contrast to the blue background of chromosomes and make the paracentromeric regions of all chromosomes but one submetacentic pair clear. Hizume et al. (1994) observed only 20 DAPI bands in L. principis-rupprechtii with two fewer at submetacentric pairs than those in our study, maybe because some DAPI bands are too faint to be identified while LPD repeats FISH signals are more obvious (Fig 1A and D). The chromosomes with similar hybridized signal size and intensity of LPD repeats could be identified as homologous pairs. Thus, in addition to the three pairs of chromosomes that have been identified by rDNA probes, other eight chromosomes pairs bearing distinct LPD repeat signals and one pair without FISH signal were found. Then the statistical analysis of microscopic measurements of
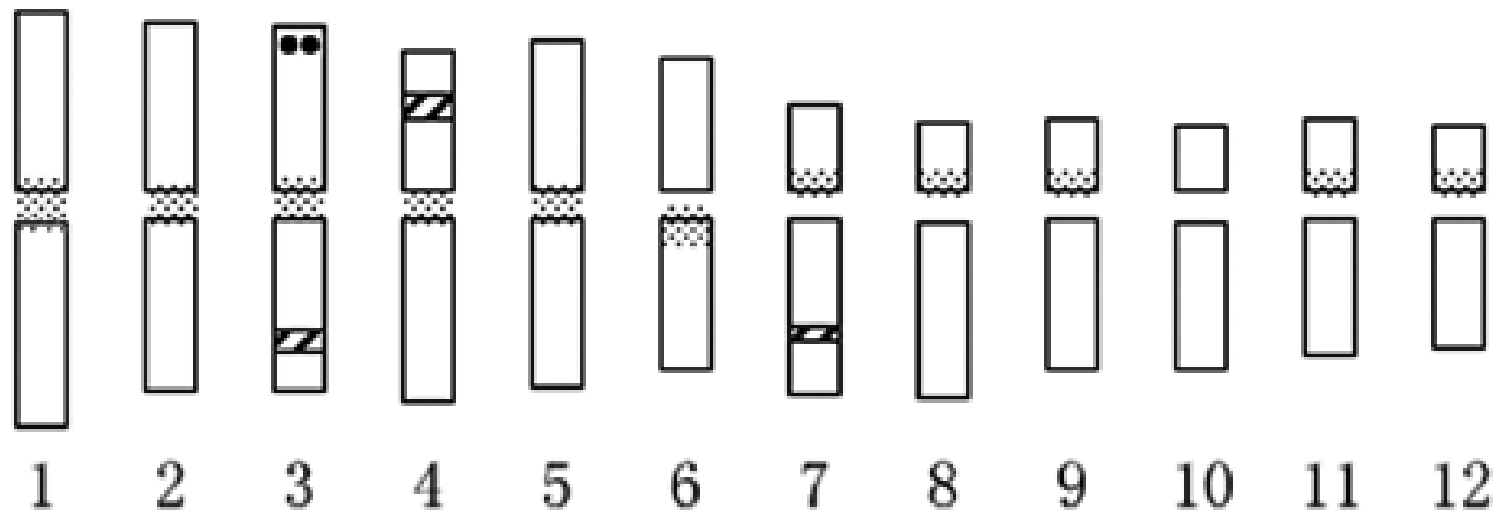

Figure 2: Idiogram of L. principis-rupprechtii with 25S rDNA (slash area), 5S rDNA (filled circle), and LPD repeat (dot) loci. 
chromosomes was carried out. Complementary chromosomes were arranged in order of decreasing length as shown in Figure $1 \mathrm{E}$ and their idiogram in Figure 2. According to the ordering, the chromosome pair bearing both $25 \mathrm{~S}$ and $5 \mathrm{~S}$ rDNA loci is No. 3. Chromosome 4 and 7 bear $25 \mathrm{~S}$ rDNA loci at their short arm and long arm, respectively. The only pair without any FISH signal is chromosome 10. The karyotype data including the length of short and long arms, arm ratio (length of long arm/length of short arm), and relative length estimated for each chromosome were listed in Table I. Since the homologous chromosome pairing is more credible with the assistance of multicolor FISH signals, the karyotype data could be more accurate than those obtained from conventional karyotype. However, the length of the chromosomes within the large group and the medium-sized group each are so close that some ordering could be mistaken. This could even happen for the identification of long/short arm of the metacentrics, especially for chromosome 2 and 3 . To solve this problem, more chromosome landmarks are needed, and then, the detailed karyotype could be constructed not only according to the length of chromosomes.
Simultaneous in situ hybridization of more than two different DNA probes to the same chromosome preparation is informative and could help us to complete the construction of detailed karyotypes of Larix, but there are some noticeable technical problems. First, it is important to ensure that the emission spectra of the probes are sufficiently well separated from each other, and from the counterstaining of the chromosomes. We selected Cy 3 and FITC as reporters, which fluoresce in the red channel and green channel, respectively. They are distinct from each other and also from the blue background of chromosomes produced by DAPI. Secondly, we should be cautious with probes whose loci are totally or in part overlapped. If two probes are supposed to be detected by a single color but they colocalize at the same loci of metaphase chromosome, they will display composite color and that will foster confusion with the third probe of combinatorial labeling. Although it is possible for more than three different probes to be detected simultaneously through combinatorial labeling with more labels, or even by varying ratios of different labels for each probe, the non-standard emission filters and higher sensitivity CCD camera are needed.

TABLE I

Measurements of somatic metaphase chromosomes of L. principis-rupprechtii $(2 \mathrm{n}=24) . m$ metacentric, $s m$ submetacentric

\begin{tabular}{lccccc}
\hline $\begin{array}{l}\text { Chromosome } \\
\text { number }\end{array}$ & $\begin{array}{c}\text { Relative length } \\
\text { of short arm }\end{array}$ & $\begin{array}{c}\text { Relative length } \\
\text { of long arm }\end{array}$ & Relative length & Arm Ratio & $\begin{array}{c}\text { Chromosome } \\
\text { type }\end{array}$ \\
\hline 1 & 5.348 & 6.113 & 11.551 & 1.124 & $\mathrm{~m}$ \\
2 & 5.017 & 5.101 & 10.118 & 1.017 & $\mathrm{~m}$ \\
3 & 4.975 & 5.101 & 10.076 & 1.025 & $\mathrm{~m}$ \\
4 & 4.216 & 5.438 & 9.654 & 1.290 & $\mathrm{~m}$ \\
5 & 4.553 & 5.017 & 9.570 & 1.102 & $\mathrm{~m}$ \\
6 & 4.089 & 4.469 & 8.558 & 1.093 & $\mathrm{~m}$ \\
7 & 2.614 & 5.270 & 7.884 & 2.016 & $\mathrm{sm}$ \\
8 & 2.150 & 5.312 & 7.462 & 2.471 & $\mathrm{sm}$ \\
9 & 2.234 & 4.511 & 6.745 & 2.019 & $\mathrm{sm}$ \\
10 & 1.981 & 4.469 & 6.450 & 2.256 & $\mathrm{sm}$ \\
11 & 2.204 & 4.089 & 6.113 & 2.020 & $\mathrm{sm}$ \\
12 & 1.981 & 3.836 & 5.817 & 1.936 & $\mathrm{sm}$ \\
\hline
\end{tabular}


In this study, we demonstrated above that in gymnosperms with large chromosomes like Larix species, combinatorial labeling of probes offers a simple, inexpensive and efficient way of discerning more chromosome targets with the same number of fluorophores in one FISH experiment. By this technique and based on several appropriate probes, we may construct FISH karyotypes of $L$. principis-rupprechtii and other Larix species, and that would provide us more detailed information for karyotype analysis and even for comparison among species.

\section{ACKNOWLEDGEMENTS}

We are thankful to Dr. Robert Hasterok for kindly providing us the plasmid containing 25S rDNA and pTa794. This work was supported in part by grant G19990160 of National Key Basic Research Program 973: "Molecular Research on Tree Improvement".

\section{REFERENCES}

BROWN GR, NEWTON CH, CARLSON JE (1998) Organization and distribution of a Sau3A tandem repeated DNA sequence in Picea (Pinaceae) species. Genome 41: 560-565

FRIESEN N, BRANDES A, HESLOP-HARRISON JS (2001) Diversity, origin, and distribution of retrotransposons (gypsy and copia) in conifers. Mol Biol Evol 18: 1176-1188

GERLACH WL, DYER TA (1980) Sequence organization of the repeating units in the nucleus of wheat which contain 5S rRNA genes. Nucleic Acids Res 11: 48514865

HASTEROK R, LANGDON T, TAYLOR S, JENKINS G (2002) Combinatorial labeling of DNA probes enables multicolour fluorescence in situ hybridization in plants. Folia Histochem Cyto 40: 319-323

HIZUME M, YAMASAKI Y, KONDO K, YANG Q, HONG D, TANAKA R (1994) Fluorescent chromosome banding in two Chinese varieties of Larix gmelinii, Pinaceae. La Kromosomo II 74: 2563-2570
HIZUME $M$, SHIBATA $F$, MATSUMOTO A, MARUYAMA Y, HAYASHI E, KONDO T, KONDO $\mathrm{K}$, ZHANG S, and HONG D (2002a) Tandem repeat DNA localizing on the proximal DAPI bands of chromosomes in Larix, Pinaceae. Genome 45: 777-783

HIZUME M, SHIBATA F, MATSUSAKI Y, GARAJOVA Z (2002b) Chromosome identification and comparative karyotypic analyses of four Pinus species. Theor Appl Genet 105: 491-497

JIANG J, GILL BS, WANG PC, RONALD PC, WARD DC (1995) Metaphase and interphase fluorescence in situ hybridization mapping of the rice genome with bacterial artificial chromosomes. Proc Natl Acad Sci USA 92: 4487-4491

LEVAN A, FREDGA K, SANDBERG AA (1964) Nomenclature for centromeric position on chromosomes. Hereditas 52: 201-220

LIU B, CHEN C, LI X, CHEN R, SONG W (2005) Physical mapping of $45 \mathrm{~S}$ rDNA to metaphase chromosomes of 30 taxonomically diverse plant species. J Horti Sci Biot 80: 287-290

LIU Z, ZHANG D, HONG D, WANG X (2003) Chromosomal localization of $5 \mathrm{~S}$ and $18 \mathrm{~S}-5.8 \mathrm{~S}-25 \mathrm{~S}$ ribosomal DNA sites in five Asian pines using fluorescence in situ hybridization. Theor Appl Genet 106: 198-204

LUBARETZ O, FUCHS J, AHNE R, MEISTER A, SCHUBERT I (1996) Karyotyping of three Pinaceae species via fluorescent in situ hybridization and computer-aided chromosome analysis. Theor Appl Genet 92: 411-416

MURRAY BG, DAVIES BJ (1996) An improved method for preparing the chromosomes of Pines and other gymnosperms. Biotech Histochem 3: 115-117

MURRAY MG, THOMPSON WF (1980) Rapid isolation of high molecular weight plant DNA. Nucleic Acids Res 8: 4321-4325

RIED T, BALDINI A, RAND TC, WARD DC (1992) Simultaneous visualization of seven different DNA probes by in situ hybridization using combinatorial fluorescence and digital imaging microscopy. Proc Natl Acad Sci USA 89: 1388-1392

SILJAK-YAKOVLEV S, CERBAH M, COULAUD J, STOIAN V, BROWN SC, ZOLDOS V, JELENIC S, PAPES D (2002) Nuclear DNA content, base composition, heterochromatin and rDNA in Picea omorika and Picea abies. Theor Appl Genet 104: 505512

UNFRIED I, GRUENDLER P (1990) Nucleotide sequence of the $5.8 \mathrm{~S}$ and $25 \mathrm{~S}$ rRNA genes and the internal transcribed spacers from Arabidopsis thaliana. Nucleic Acids Res 18: 4011

VISCHI M, JURMAN I, BIANCHI G, MORGANTE M (2003) Karyotype of Norway spruce by multicolor FISH. Theor Appl Genet 107: 591-597

ZHANG X, ZHUO L, LI M (1985) A study of karyotypes of species in Larix. Hereditas (Beijing) 7: 9-11 (in Chinese) 